\title{
Estudio etnográfico sobre conocimientos cotidianos y escolares en escuelas rurales de Uruguay
}

\section{Ethnographic study on everyday and school knowledge in rural schools in Uruguay}

\section{Estudo etnográfico do saber cotidiano e escolar em escolas rurais do Uruguai}

Daniela Carolina Diaz Santos ORCID ID: $000000031837-1101$ Universidad de la República, Uruguay

Autor referente: danpsi2@gmail.com

Historia editorial:

Recibido: 22/10/2020

Aceptado: 18/02/2021

\section{RESUMEN}

Se expone un estudio en dos escuelas rurales de Uruguay cuyo objetivo fue identificar modos de presentación de los conocimientos cotidianos en el aula y describir prácticas de enseñanzas que los consideren y los relacionen con los escolares desde el marco interpretativo del constructivismo y la psicología cultural. Se seleccionaron dos escuelas unidocentes cercanas entre sí y con características similares en relación a entorno geográfico y características socioproductivas de las familias, pero con la diferencia de que en una de ellas se presentaba un proyecto de centro vinculado a las actividades productivas rurales. La metodología de investigación fue cualitativa por medio del método etnográfico, utilizando como técnicas de recolección de la información las observaciones y las entrevistas tanto a niños como a maestras. Se construyeron las siguientes categorías de análisis: características y condiciones de aparición y presentación de los conocimientos cotidianos; prácticas de enseñanza que toman en consideración los conocimientos cotidianos y las 
relaciones entre conocimientos cotidianos y escolares establecidas en el aula. Los resultados destacan que los conocimientos cotidianos se encuentran presentes de diferentes modos, ya sea por presentación espontánea de los niños o por prácticas de enseñanza docentes que fomentan relaciones con diferentes niveles de complejidad. Ello permite concluir que, a pesar de las diferencias en las actividades de enseñanza, ambas escuelas funcionan como comunidades de práctica donde se negocian significados.

Palabras claves: Conocimiento cotidiano; conocimiento escolar; escuelas rurales.

\section{ABSTRACT}

A study in two rural schools in Uruguay is presented. Its objective was to identify ways of presenting everyday knowledge in the classroom and describe teaching practices that consider them and relate them to schoolchildren from the interpretive framework of constructivism and cultural psychology. Two nearby singleteacher schools were selected with similar characteristics in relation to geographic environment and socioproductive characteristics of the families, but with the difference that in one of them a center project linked to rural productive activities was presented. The research methodology was qualitative through the ethnographic method, using observations and interviews with both children and teachers as information gathering techniques. The following categories of analysis were constructed: characteristics and conditions of appearance and presentation of everyday knowledge; teaching practices that take into account everyday knowledge and the relationships between everyday and school knowledge established in the classroom. The results highlight that everyday knowledge is present in different ways, either by spontaneous presentation of children or by teaching practices that foster relationships with different levels of complexity. This allows us to conclude that, despite the differences in teaching activities, both schools function as communities of practice where meanings are negotiated.

Keywords: Everyday knowledge; school knowledge; rural schools.

\section{RESUMO}

Apresenta-se um estudo realizado em duas escolas rurais do Uruguai, cujo objetivo foi identificar os modos de apresentação dos conhecimentos cotidianos em sala de aula, e, a partir de um marco interpretativo do construtivismo e a psicologia cultural, descrever as práticas pedagógicas que consideram e relacionam os conhecimentos escolares com os 
conhecimentos cotidianos. Foram selecionadas duas escolas unidocentes, próximas entre si, com características similares em relação ao entorno geográfico e características sócioprodutivas das famílias, porém com a diferença de que uma delas apresentava um projeto de centro vinculado às atividades produtivas rurais. A metodologia da pesquisa foi qualitativa, por meio do método etnográfico, utilizando como técnica de coleta de dados, observações e entrevistas com as crianças e professoras. Construíram-se as seguintes categorias análises: características e condições de surgimento e apresentação dos conhecimentos cotidianos; práticas pedagógicas que levam em consideração os conhecimentos cotidianos e as relações entre conhecimentos cotidianos e escolares estabelecidos na sala de aula. Os resultados destacam que os conhecimentos cotidianos se encontram presentes de diferentes modos, tanto pela apresentação espontânea das crianças, como pelas práticas pedagógicas que fomentam relações com diferentes níveis de complexidade. Isso permite concluir que, a pesar das diferenças nas atividades de ensino, ambas as escolas funcionam como comunidades de prática onde se negociam significados.

Palavras-chave: Conhecimento cotidiano; conhecimento escolar; escolas rurais.

$\mathrm{D}$ esde hace varias décadas se viene considerando la escuela rural como una institución con visión propia de la cultura dominante y urbana y por lo tanto desvalorizante de los conocimientos de las familias y las comunidades, lo que establece una brecha con el conocimiento escolar que lleva a reproducir las desigualdades sociales (Borsotti, 1984; Martín-Barbero, 1999; Gajardo, 2014). En la década del 90, aportes constructivistas y de la psicología cultural estudiaron contextos específicos de enseñanza, rescatando por ejemplo, el valor de los saberes campesinos familiares (Moll y Greenberg, 1993).Por otra parte, estudios etnográficos como los de Rockwell (1997) en escuelas rurales de México, plantearon que a menudo son menores las distancias entre los programas y las prácticas educativas que lo que se supone. Recientemente, varios estudios sobre los conocimientos campesinos, también denominados desde la etnoecología como conocimientos ecológicos locales 
(Almendra, Peña-Cortés y Rojas Maturana, 2011; Riat, 2016; Melo Brito, 2020), procuran rescatarlos no solo entendiendo que pertenecen a sujetos cuya identidad debe ser respetada, sino que aportan a los problemas socioambientales de la actualidad. En Uruguay existe una vasta tradición histórica en el campo de la pedagogía sobre escuela rural (Soler, 1996) y en los últimos años se destacan estudios sobre el multigrado desde las ciencias de la educación que dan cuenta de la especificidad didáctica que tiene la escuela rural así como de la importancia de tomar en cuenta los recursos del medio para la enseñanza de los contenidos (Santos, 2011). Sin embargo, desde el ámbito de la psicología de la educación y específicamente la psicología cultural, no se han encontrado estudios sobre la construcción del conocimiento y el lugar de los saberes cotidianos en las escuelas rurales uruguayas. El estudio etnográfico del que da cuenta el presente artículo permitió identificar características y modos de presentación de los conocimientos cotidianos, así como las prácticas de enseñanza que los consideraban y las relaciones con los conocimientos escolares en dos escuelas rurales uruguayas.

\section{Los conocimientos cotidianos}

En la psicología educacional y cultural los conocimientos cotidianos son conocimientos legítimos en lo referente a la resolución de problemas en la vida cotidiana y brindan sentidos a la realidad concreta en la que los sujetos se encuentran inmersos (Rodrigo, 1997; Delval, 2000). Los conocimientos aprendidos en la vida cotidiana o en espacios informales permiten a los sujetos formar parte de la cultura de su comunidad al interactuar con miembros más expertos (Del Río, 1992; Lacasa, 1994; Rogoff, 1993). Moll y Greenberg (1993) utilizan el concepto de fondo de conocimiento para referir a cuerpos de conocimiento específicos de importancia estratégica para las familias: habilidades, información, valores y normas culturales, transmitidos de generación en generación. Para Rogoff (1993) en comunidades como las rurales, los sujetos desde 
pequeños participan precozmente con sus padres en tareas económicas y domésticas obteniendo una educación informal que le brinda conocimientos sobre agricultura, ganadería, artesanía y otros aspectos que le serán útiles en la vida rural.

\section{El conocimiento escolar y sus relaciones con el conocimiento cotidiano}

Vigotsky (1982) fue pionero al definir y caracterizar los conceptos científicos, aquellos adquiridos mediante la escolarización y los conceptos espontáneos o cotidianos aprendidos en entornos informales. Los conceptos científicos tienen como características la definición verbal primaria, la abstracción, la insuficiente saturación de lo concreto y el hecho de que son aprendidos en un ambiente de escolarización. Los espontáneos en cambio son aprendidos en entornos informales, concretos, incapaces de abstracción y poseen una escasa posibilidad de ser definidos verbalmente. Unos y otros a pesar de sus raíces genéticas diferentes interactúan de modo que los conceptos abstractos descienden hacia niveles más concretos y los espontáneos ascienden hacia niveles de mayor formalización. Para el autor, el desarrollo de los conceptos científicos depende de un conjunto de conceptos cotidianos preexistentes. Desarrollos posteriores (Pozo, 1998) plantean que la opción adecuada sería promover una diferenciación e integración jerárquica entre distintos tipos de conocimientos y concebirlos como niveles alternativos de análisis de un mismo problema. Para las perspectivas de aprendizaje situado el conocimiento cotidiano en la escuela debe ser tomado como una entidad en sí misma potenciando ciertas relaciones con el escolar pero sin perder el sentido, la identidad y el ámbito de aplicación de cada uno. De ahí que el conocimiento cotidiano brinde fuerza motivacional al escolar y éste lecturas alternativas que complejicen la visión cotidiana del mundo (Rodrigo, 1997). 


\section{Prácticas de enseñanza en la escuela rural}

Rockwell (1997) plantea que existen formas heterogéneas de construcción cotidiana de los conocimientos, significados y prácticas en el contexto escolar donde el conocimiento local penetra en la escuela mediante las prácticas y los discursos de los maestros y alumnos, lo que lleva a reinterpretaciones y negociaciones sobre los contenidos y las normas escolares. De esta manera la escuela se convierte en una comunidad de práctica donde los que aprenden participan activamente y comparten las metas de aprendizaje (Lacasa, 1997). En Uruguay, algunos pedagogos han reflexionado en profundidad sobre cómo debe funcionar la escuela rural para garantizar aprendizajes auténticos de la cultura de las comunidades. Un referente ha sido Soler (1996) quien plantea la necesidad de una pedagogía del medio valorizando una enseñanza que tome en cuenta los intereses y las necesidades de los alumnos. De este modo, es necesario "apoyarse en lo concreto, lo conocido, lo suyo ya aprehendido" y afirma que lo que es importante es "crear auténticas situaciones de aprendizaje motivadas en la realidad" (p. 145).

\section{Metodología}

El estudio tuvo como objetivos fundamentales identificar características y modos de presentación de los conocimientos cotidianos y analizar las prácticas escolares que los consideran, así como las relaciones con los conocimientos escolares en dos escuelas rurales del sureste del Uruguay. Se realizó por medio de una metodología cualitativa, específicamente a través del método etnográfico, entendiendo que era el adecuado para la comprensión de lo que acontece en las aulas al permitir la inclusión del investigador en la cotidianeidad de la escuela como observador de las actividades e intercambios. Desde ese marco se realizó un estudio de caso múltiple (dos escuelas) logrando un análisis sistemático de los escenarios educativos a lo largo de un año lectivo. En el estudio desarrollado se seleccionaron dos escuelas rurales pero en base 
a un criterio diferenciador: la presencia en una de ellas de un proyecto de centro relacionado a actividades rurales.

\section{Participantes}

Participaron del estudio niños/as y maestras de dos escuelas rurales del sureste del Uruguay. En el artículo se presentan nombres ficticios para preservar la identidad de los participantes. Se destaca el carácter unidocente de las escuelas y su funcionamiento en multigrado. Ambas escuelas se encuentran ubicadas en una zona agrícola ganadera del sureste uruguayo con población rural dispersa, básicamente constituida por pequeños productores y asalariados rurales mediando una distancia de 10 kilómetros aproximadamente entre ellas. La escuela que se denominará a partir de ahora Escuela Granja era categorizada como Escuela Granja Experimental, siendo su maestra (Alicia) efectiva en el cargo desde hacía quince años y desarrollando un proyecto de escuela inspirado en la propuesta de escuela productiva del programa de escuelas rurales de 1949 ( CNEPyN., 1950). La institución contaba con un funcionario específicamente para las tareas productivas que guiaba las actividades con los niños/as en coordinación con la maestra. La segunda escuela llamada Escuela Ruta no presentaba en términos formales un proyecto curricular específico relacionado a actividades del medio. Su maestra (Rosana) era efectiva desde hacía ocho años en el cargo.

Como técnicas se utilizaron observaciones de carácter etnográfico en la escuela y entrevistas formales a maestras e informales a maestras y niños/as como complemento de las observaciones durante períodos de dos años. A través de una guía de campo que permitió estructurar la observación en determinadas líneas, se realizaron observaciones de actividades planificadas y libres, y de situaciones en diferentes espacios y momentos escolares. 


\section{Análisis de la información}

Se intercalaron períodos de observación con períodos de análisis y lectura teórica o temática y periodos de elaboración conceptual y reelaboraciones de categorías, las cuáles se fueron sucesivamente construyendo de acuerdo al método etnográfico (Rockwell, 2009). Dichas categorías se definieron en el estudio como: características y condiciones de aparición y presentación de los conocimientos cotidianos; prácticas de enseñanza que tomaran en consideración los conocimientos cotidianos y las relaciones entre conocimientos cotidianos y escolares establecidas en las actividades escolares.

\section{Resultados}

Características y condiciones de presentación de los conocimientos cotidianos.

El estudio se propuso entre sus objetivos identificar cómo se presentaban los conocimientos cotidianos en las escuelas rurales seleccionadas. Como resultado principal se destaca que estos mostraron diversas formas de aparición y presentación en ambas instituciones. Por un lado, se identificó en las dos escuelas un surgimiento espontáneo de los conocimientos cotidianos por parte de los niños a modo de presentación frente al investigador y expresión de identidad: "Hoy anduve a caballo" (Santiago, 6 años, Escuela Granja, 2011); "Sabés que en casa estoy haciendo un embarcadero con un amigo, nos llevó varios días hacerlo" (Nicolás, 6 años, Escuela Ruta, 2011); "Con mis padres planté tomates y melones... me gustan, pero me encantan las sandías" (Sandra,10 años, Escuela Granja, 2011). Específicamente en la Escuela Ruta pudieron identificarse conocimientos cotidianos presentes en relatos de anécdotas que se dan entre niños o entre niños y maestra en condiciones específicas como los recreos, la entrada a la escuela, en el comedor o cuando la maestra no se encontraba en el salón. Las siguientes conversaciones sucedieron en un momento que la misma salió del espacio de aula. Ricardo: "Anduve el fin de semana con mi padre en 
el tractor, a caballo también". Nicolás: "Yo sé andar en tractor". Camila: "El otro día me caí del caballo pero no me pasó nada". (Escuela Ruta, 2011)

Existieron por otra parte situaciones puntuales donde la docente promovió el intercambio sobre vivencias cotidianas como la de la siguiente conversación iniciada por ella a comienzo de semana:

Maestra: ¿Cómo pasaron el fin de semana?

Nicolás: Nosotros armamos una hamaca y nos caímos.

Maestra: - ¡Qué peligro! Tiene que haber una persona mayor que supervise.

Además tiene que estar bien atada, que no hayan piedras debajo.

Nicolás: Sí, no nos quedó bien atada.

Maestra: ¿Y los demás?

Sofía: Yo planté boniatos con mi padre.

Ana: Yo jugué al restaurante con estos dos haciendo tortas de barro.

Maestra: ¡Y se llenaron de energía para hacer los trabajos!

Todos los niños: ¡Noo! (Díaz, 2012)

Conversaciones de este tipo no fueron consideradas para ninguna tarea escolar, sino por su función el simple intercambio personal con los otros y por la necesidad de conocer a sus alumnos y saber cómo pasaron. Al respecto, en una entrevista la maestra expresaba: "Me gusta saber qué hicieron el fin de semana, me parece que tiene que ver con el vínculo, es un rato, porque hay mucho para trabajar". (Rosana, Escuela Ruta, 2011).

En lo que refiere a la aparición de conocimientos cotidianos en la Escuela Granja se destacan las siguientes situaciones: diálogos que muchas veces no eran escuchados por la maestra dentro del salón de clase cuando los niños terminaban la tarea; comentarios espontáneos entre niños y/o entre niños y maestra al estar realizando una actividad y comentarios y anécdotas durante la realización de tareas productivas fuera del salón de clase. El siguiente diálogo sucedió entre dos niños pequeños que 
terminaron una tarea en el cuaderno de clases y no tuvo relación con la misma: Santiago: "Tengo una perra, juego con ella, se me mete abajo de las patas".Manuel: "Tené cuidado con las pulgas y garrapatas, tenés que lavarla, darle una ducha". (Díaz, 2011). Por otro lado aparecían anécdotas y conversaciones sobre temas cotidianos como consecuencia de una tarea de clase formal relacionada a los mismos. Ello puede ser considerado como una manifestación espontánea por parte de los niños ya que no era intencionalmente previsto por la maestra como en el siguiente ejemplo registrado en diario de campo:

Los niños están realizando una producción de texto sobre lo realizado el día anterior en el invernáculo de la escuela y surge el comentario de algunos con respecto a los invernáculos que tienen en la casa y plantean lo siguiente: Sandra: Las plantas de tomates que plantamos con mamá y papá se pusieron feas, se la comieron unos bichos, vamos a plantar otros. Nelson: Nosotros vendimos el otro día el primer cajón de tomates. (Díaz, 2012) En este caso, la tarea escolar al referir a temas vinculados al trabajo en la huerta generaba la narración de experiencias cotidianas en el hogar lo que permitiría ver cierta continuidad entre los escenarios cotidianos y los escolares. Es importante destacar que otro modo de expresión de los conocimientos cotidianos específico de la Escuela Granja y que pudo visualizarse fue la acción o actividad práctica del niño. Ello se debía al formato escolar de la escuela, donde las actividades productivas ocupaban un lugar fundamental y el niño era partícipe directo. Dichas actividades, similares en la casa y en la escuela, permitían que se pusieran en juego el conocimiento cotidiano, en términos de conocimiento práctico, ligado a la acción y en relación al uso de herramientas. La siguiente transcripción del diario de campo muestra ello:

Los niños agarran sus gorros y vamos hacia la quinta donde las plantas de boniatos ya fueron trasplantadas. Algunos niños por turno van agarrando azadas y empiezan a carpir. Algunos lo hacen con una habilidad sorprendente, 
incluso la niña pequeña de 5 años (Cecilia), que me dice: "Yo carpo en casa con mamá". La maestra Alicia manifiesta: "te das cuenta clarito de los que saben carpir porque en la casa lo hacen, son los que van más rápido y no dañan la planta". (Díaz, 2012)

En síntesis, las narrativas donde se presentaban conocimientos cotidianos daban cuenta de la acción y del saber-hacer y como características fundamentales se destacó el referir básicamente a conocimientos vivenciales o sobre el mundo físico y ser poco permanentes en el tiempo, excepto que una intervención intencionada de la maestra fomentara un mayor desarrollo.

\section{Prácticas docentes que favorecen la aparición de conocimientos cotidianos}

Por otro lado, en el estudio se pretendía identificar prácticas de enseñanza que consideraran los conocimientos cotidianos de los niños y sus familias. Como resultados al respecto se destaca que en ambas escuelas las propuestas de las maestras incorporaban la dimensión de la cotidianeidad del niño rural, pero de modo diferente. En la Escuela Ruta si bien la docente manifestó utilizar escasamente el conocimiento cotidiano, hacía una selección de actividades y propuestas que implícitamente mostraban negociaciones dentro de una comunidad de aprendizaje procurando la motivación de los niños. Ello se observó con mayor frecuencia en el contenido de algunas actividades en matemática, lengua y ciencias naturales. En este sentido, la maestra respondió frente a la pregunta de cuáles eran las tareas en las que introducía conocimientos cotidianos: "En la oralidad, la producción de texto, razonamiento, que sean algo de lo que ellos manejan. Si le pones un trabajo de razonamiento que no tiene nada que ver con lo que hacen todos los días... cuesta más" (Rosana, Escuela Ruta, 2012). A modo de ejemplo se mencionan las siguientes actividades: "Redacta: cómo se hace un huevo frito" (actividad domiciliaria de lengua); "Redacta: Qué sabes de las abejas" (actividad domiciliaria de lengua y ciencias 
naturales); "Resuelve: Son 2350 caballos, corren 3/9 en la próxima carrera, ¿cuántos corren?" (actividad de clase de matemática). Frente a la pregunta de la investigadora acerca de si las características de la zona y el entorno eran usados como recursos para contenido de tareas, la maestra planteó: "Sí, por ejemplo en matemática, se pone algo sobre la leche y que tengan que hacer algo, se resuelve más fácil. Cuando trabajas la centena, si lo decís con billetes sale más fácil, es lo más cercano de ellos" (Rosana, Escuela Ruta, 2012). De este modo la maestra da cuenta del valor de la contextualización para la resolución de tareas escolares. En segundo lugar podemos afirmar que actividades prácticas realizadas en alguna oportunidad en la escuela como medir un alambrado, visitar un tajamar y mencionadas por los niños y niñas como significativas, tenían un carácter excepcional siendo escasamente visualizadas. Algunas situaciones pertenecientes al escenario rural, pero relacionadas con la escuela, como la presencia de vacunos como insumo para recaudar dinero o sucesos que captaban la atención de los niños (presencia de un lagarto y abejas en la escuela por ejemplo), no fueron visualizados como recursos en las actividades de la maestra. En cuanto a la Escuela Granja se destaca que de acuerdo al formato escolar diseñado por la maestra se trataba que los contenidos programáticos se relacionaran con las actividades productivas y la naturaleza. En ese sentido mencionó en entrevista:

En la parte rural nos marca la naturaleza, la esquila es ahora, el nacimiento de un cordero mañana y en el programa capaz que está más adelante, no puedo llevar la naturaleza al programa... Uno le da la adecuación, no nos tenemos que sujetar al programa, no hice una planificación lineal. Ellos vienen con vivencias y trabajás, uno trajo una liebre y trabajo la observación y eso te da pie, no tengo problema en cambiar todo lo que planifiqué para ese día (Alicia, Escuela Granja, 2012). 
Se puede afirmar que el entorno penetraba en la escuela al ser reconocida la propia naturaleza como el escenario y el contenido de las actividades escolares. A su vez las vivencias y conocimientos de los niños se convertían en situaciones de aprendizaje auténticas en el aula, ya que en ocasiones eran considerados como métodos de resolución de una tarea o utilizados para trabajar en oralidad. Se mencionan algunas actividades a modos de ejemplo: conjugación de verbos asociados a tareas realizadas en la escuela, por ejemplo, el verbo "plantar"; recetas con producciones de textos acompañados de su realización práctica en la cocina de la escuela (actividades de lengua). En matemática se planteaban problemas y razonamientos sobre la recolección de huevos o sobre la cosecha y venta de hortalizas realizada en la escuela —desde simples sumas en primer año escolar hasta porcentajes en sexto año—. Se destaca además que las actividades de granja eran realizadas en grupo bajo la guía de la maestra y del funcionario escolar, quien participaba directa o indirectamente brindando niveles mayores de autonomía de acuerdo a la edad de los niños. Eran actividades frecuentes la recolección de huevos, trabajo en invernáculo con diferentes hortalizas; cuidado de árboles frutales, cultivo de lombrices californianas, cría de cerdos, cría y cuidado de lanares, y actividad de tambo con vacunos. Un aspecto a destacar es que ambos escenarios, el de granja y el de clase, estaban íntimamente interconectados, dado que lo que se trabajaba en clase tenía que ver en gran parte con lo realizado en el espacio de la granja como lo expresaba la maestra Alicia:

Preparamos dulce de leche, lo medimos, lo preparamos y al otro día lo comimos... para ellos era el mejor dulce de leche. Eso es contextualizar, aprovecho todo, la medición, proporción, física, movimiento... si no tuviera la parte agronómica no sé si sabría trabajar en una escuela (Alicia, Escuela Granja, 2012). 


\section{Las relaciones que se establecen entre los conocimientos cotidianos y los} escolares

En relación a diferentes tipos de relaciones presentes en ambas escuelas entre conocimientos cotidianos y escolares, se destaca la heterogeneidad y complejidad presentes en ambas superando simples relaciones como podría ser el tomar el conocimiento cotidiano como punto de partida para enseñar un contenido escolar. Se referirá en el presente artículo a los niveles de relaciones considerados significativos y se ejemplificarán los mismos. En relación a la Escuela Ruta se visualizó un nivel que refiere a conocimientos cotidianos como recursos didácticos para los escolares. En este caso, la maestra tomaba conocimientos e intereses del mundo cotidiano del niño como apoyo para la formulación de consignas como los problemas en matemáticas que ya fueron mencionados en el apartado anterior en el entendido que generaban motivación y mejores resoluciones. Otro nivel lo constituye la relación entre conocimientos cotidianos y actividades de escritura en producciones libres donde el niño podía elegir la temática a desarrollar. Los niños pasaban a un plano simbólico vivencias, experiencias y conocimientos que eran activados con las propuestas de este carácter. Un ejemplo frecuente eran actividades escritas bajo la consigna: "Cuenta que hiciste en vacaciones". Finalmente, se destaca el uso de conocimientos cotidianos como ejemplos concretos de conceptos científicos y pudo visualizarse en actividades donde el niño ejemplificaba con elementos pertenecientes a su mundo cotidiano (que él mismo conoce por experimentación, vivencia u observación) los conceptos aprendidos en la escuela lográndose un enriquecimiento mutuo de ambos conocimientos. Se ejemplifica este nivel con una actividad observada de ciencias naturales sobre proteínas, glúcidos y lípidos. En una primera etapa los niños debían copiar un esquema del pizarrón sobre los conceptos a modo de definiciones. En un segundo momento se solicitaba que los niños en grupo escribieran ejemplos de alimentos que formaran parte de su vida cotidiana clasificándolos en las tres 
categorías. De este modo, el concepto abstracto podía comprenderse gracias al ejemplo concreto del conocimiento cotidiano y este se enriquecía en tanto adquiría un sentido diferente: algunos alimentos permiten reponer energía, otros favorecen la formación de huesos y tejidos por ejemplo.

En relación a la Escuela Granja se pudieron identificar niveles de relaciones entre conocimientos cotidianos y escolares con mayor complejidad. Por un lado se visualizaron situaciones escolares a resolver donde el problema era cotidiano pero por el solo hecho de estar presente en la escuela el niño lo resolvía escolarmente. De este modo se generaban representaciones escolares para situaciones cotidianas. Por ejemplo, se les preguntó a los niños si las actividades que hacían en la escuela se parecían a las realizadas en el hogar. Un niño de 9 años respondió:

Se parece lo de los animales, se parece a casa, la esquila, castrar, pero es diferente como se hace; en casa no se usa gomita, se hace con cuchillo, acá cuidan más a los animales... un día mi padre mató un ternero y lo dejo un rato largo que se muera...(Nicolás, Escuela Granja, 2012).

Se reconocía un sentido diferente a la actividad realizada en la escuela conviviendo dos formas de resolver la tarea. Otro nivel de relaciones consistía en considerar al conocimiento cotidiano como método válido de resolución de ciertas tareas. Ante la pregunta “¿Qué lugar ocupan los conocimientos cotidianos en tu práctica?” la maestra Alicia planteaba:

Es una integración que hay que aprovechar, los gurises pueden saber más que uno... Nosotros aprendimos con un alumno cómo plantar papas, la papa grande para aprovecharla, la cortaban a la mitad y la sellaban, lo experimentamos y ahora lo usamos. Si te pones a analizar costumbres, ejemplos, usamos lo de perejil en menguante $\mathrm{Y}$ es una creencia, es rescatable. Alguna explicación científica tiene que tener, hemos plantado para comprobar si es cierto....(Alicia, Escuela Granja 2012). 
Coexistían entonces, formas de resolver problemas que no necesariamente eran diferentes en uno u otro ámbito, es decir que no siempre un mismo tema o situación debía resolverse de modo más elaborado en el ámbito escolar. El contexto escolar permitía e incluso fomentaba una forma de resolución cotidiana. Aquellos conocimientos vinculados a la actividad práctica como el uso de herramientas en el trabajo en la huerta por ejemplo, se veía facilitado porque era conocido por los niños al participar de tareas similares en los hogares. Por otra parte se identificaron en las actividades escolares, explicaciones "científicas" a situaciones cotidianas. En este caso se mantenía la temática común cotidiana tanto en la escuela como en el hogar pero con la diferencia de que en la escuela se busca la comprensión científica de la realidad cotidiana. La maestra planteaba que las actividades rurales que se llevaban a cabo en la escuela podían realizarse de forma diferente al hogar mostrando medios más eficaces y elaborados o realizarse de la misma forma pero siendo tarea de la escuela trabajar con los alumnos las "explicaciones científicas" de por qué se realizaban de esa manera o para qué. En relación a la castración en lanares por ejemplo mencionaba:

Usar gomita ( consiste en atar fuertemente los testículos de los lanares con una goma de forma que con el paso de los días los mismos se secan y caen), es una estrategia más higiénica menos agresiva que el corte, además hablamos en la escuela de por qué castrar, desde el manejo del campo, producción y parte genética, la explicación científica que es impedir la reproducción, importa verbalizar el por qué, porque en la casa no le dicen que los castramos para que no se produzcan espermatozoides, para que no fecunden a las hembras, eso es la tarea de la escuela. (Alicia, Escuela Granja, 2012).

En relación a ello planteaba uno de los niños: "Les enseñamos a los niños de la ciudad cómo se castra; lo hicimos con gomita, no con cuchillo, y explicamos por qué y le dijimos que con cuchillo se hacía a veces en las casas nuestras" (Francisco, 9 años, 
2012). En este caso, el método que se usaba en la escuela, más higiénico y menos agresivo se acercaría más al conocimiento científico y era priorizado en la escuela frente a la acción más tradicional llevada a cabo en los hogares. Por otro lado se identificaron relaciones entre conocimientos escolares y cotidianos en términos de transferencia de aprendizaje en tanto se podía hacer en casa lo que se hacía en la escuela. Por medio de una Fundación, la Escuela Granja transmitió las ventajas de los invernáculos y se brindaron ayudas específicas a las familias para construir los propios. En este caso la enseñanza escolar no solo no separaba a los niños de su vida cotidiana familiar, sino que aportaba un conocimiento de utilidad para los hogares. Por último, un nivel identificado fue el de la presencia de conocimientos científicos en escenarios cotidianos reales específicamente vinculados a la matemática. Se visualizaron actividades matemáticas insertas en contextos de resolución real de problemas, como las realizadas en el galpón escolar, el cual oficiaba también como un aula. En ese espacio se pesaban y empaquetaban arvejas, se guardaban los huevos en los cartones, se clasificaba y pesaba la lana de la esquila, etc. La presencia de un pizarrón en el lugar facilitaba la tarea ya que los niños bajo la tutoría de la maestra anotaban datos y realizaban diferentes razonamientos.

\section{Reflexiones finales}

El estudio dio cuenta de las diversas presentaciones del conocimiento cotidiano y las múltiples formas de interrelacionamiento con el escolar, aportando elementos teóricos sobre las características del conocimiento y su apropiación en la escuela rural. A pesar de los planteamientos clásicos sobre la escuela rural reproductora de la cultura dominante, se concluyó que las escuelas rurales estudiadas funcionaban como comunidades de práctica donde los conocimientos cotidianos se encontraban presentes y eran de un modo $u$ otro considerados. En ese sentido, las narraciones de los niños pudieron ser consideradas como el vehículo privilegiado para conocer los 
conocimientos cotidianos y las prácticas de enseñanza que incorporaban algunos aspectos de los mismos parecieron propiciar la motivación por el aprendizaje. En la Escuela Ruta si bien no existía formalmente un currículo referido a las actividades rurales, los conocimientos cotidianos estaban presentes y eran considerados principalmente como contenido de las actividades escolares. De todos modos, mucho de lo acontecido en la vida familiar o incluso en la vida escolar no era considerado en propuestas concretas o en intervenciones espontáneas por parte de la docente. En la Escuela Granja donde las propuestas eran actividades productivas como parte del currículo se manifestaba claramente una continuidad con los saberes y prácticas del hogar y las relaciones entre conocimientos visualizadas adquirieron mayores niveles de complejidad. Se puede considerar que propuestas didácticas que incorporen la dimensión de los conocimientos cotidianos pero desde un lugar que amplíe la estrategia de "contextualización" permitirían superar la brecha entre los intereses de los niños y lo que la escuela les ofrece. En este sentido, la negociación de significados parece ser un factor fundamental para lograr una auténtica apropiación y democratización del conocimiento. Por último se destaca que la posibilidad de replicación de este estudio puede permitir identificar particularidades de comunidades de prácticas en diferentes contextos geográficos y socioculturales y conocer entonces, los saberes cotidianos de niños y familias.

\section{Referencias}

Almendra, O., Peña-Cortés F., y Rojas Maturana, M. (2011). Relación entre saberes y conocimientos territoriales en escuelas Lafkenches: la necesidad de un currículum intercultural con base geográfica. EXE, 10(20), 35-58. Recuperado de http://www.rexe.cl/ojournal/index.php/rexe/article/view/109

Borsotti, C. (1984). Sociedad rural, educación y escuela en América Latina. Buenos Aires: Kapelusz. 
CNEPyN. (1950).Programa para escuelas rurales. Montevideo: Imprenta Nacional.

Del Río, P. (1992). El niño y el contexto sociocultural. Anuarios de Psicología, 53, 61 69.

Delval, J. (2000). Aprender en la vida y en la escuela. Madrid: Morata.

Gajardo, M. (2014). Educación y desarrollo rural en América Latina. Reinstalando un campo olvidado de las políticas educativas. Revista Iberoamericana de Evaluación Educativa, 7(3), 15-27. Recuperado de https://revistas.uam.es/index.php/riee/article/view/3099

Lacasa, P. (1994). Aprender en la escuela, aprender en la calle. Madrid: Visor.

Lacasa, P. (1997). Construir conocimientos: ¿saltando entre lo científico y lo cotidiano?. En M. Rodrigo y J. Arnay (Comps). La construcción del conocimiento escolar (pp. 81-106). Barcelona: Paidós.

Martín-Barbero, J. (1999). Las transformaciones del mapa cultural: una visión desde América Latina. Ámbitos. Revista internacional de comunicación, 2, 7-21. Recuperado de https://idus.us.es/handle/11441/67269

Melo Brito, N. (2020). Puentes entre conocimientos científicos escolares y conocimientos ecológicos tradicionales: un estudio de aula en la comunidad wayuu (Tesis de doctorado, Facultad de Ciencias y Educación, Universidad Francisco José de Caldas, Bogotá). Recuperado de http://hdl.handle.net/11349/24851

Moll, L., y Greenberg, J. (1993). Creación de zonas de posibilidades: combinación de contextos sociales para la enseñanza. En L. Moll (Comp.), Vygostky y la educación (pp. 371-403). Buenos Aires: Aique.

Pozo, J. (1998). Del conocimiento cotidiano al conocimiento científico: más allá del cambio conceptual. En J. Pozo y M. Gómez. Aprendizaje y Enseñanza de las Ciencias (pp. 128-146). Madrid: Morata. 
Riat, P. (2016). Pequeños recorridos, grandes saberes: el conocimiento ecológico local compartido por niños y adolescentes en una escuela rural de Santiago del Estero, Argentina. Bonplandia, 25(2), 87-102. Recuperado de https://www.researchgate.net/publication/312922620

Rockwell, E. (1997). La dinámica cultural en la escuela. En A.ALvarez (Ed.)Hacia un curriculum cultural. La vigencia de Vigostky en la educación (pp. 21-38). Madrid: Fundación Infancia y Aprendizaje.

Rockwell, E. (2009). La experiencia etnográfica. Buenos Aires: Paidós.

Rodrigo, M.J. (1997). Del escenario sociocultural al constructivismo episódico: un viaje al conocimiento escolar de la mano de las teorías implícitas. En M. Rodrigo y J. Arnay (Comps). La construcción del conocimiento escolar (pp. 177-191). Barcelona: Paidós.

Rogoff, B. (1993). Aprendices del pensamiento. El desarrollo cognitivo en el contexto social. Barcelona: Paidós.

Santos, L. (2011). Aulas multigrado y circulación de los saberes: Especificidades didácticas de la escuela rural. Profesorado. Revista de curriculum y formación del profesorado, 15(2), 71-91. Recuperado de https://www.ugr.es/ recfpro/rev152ART5.pdf

Soler, M. (1996). Educación y vida rural en América Latina. Montevideo: Federación Uruguaya de Magisterio - Instituto del Tercer Mundo.

Vigotsky, L. (1982). Estudio del desarrollo de los conceptos científicos en la edad infantil. En L. Vigotsky Obras Escogidas // (pp. 181-285). Madrid: Visor.

\section{Declaración de contribución de autores}

DD contribuyó en la totalidad del artículo. 


\section{Editor de sección}

La editora de sección de este artículo fue Pilar Bacci.

ORCID ID: 0000-0002-6611-1905

Formato de citación

Diaz Santos, D.C. (2021). Estudio etnográfico sobre conocimientos cotidianos y escolares en escuelas rurales de Uruguay. Psicología, Conocimiento y Sociedad, 11(1), 177-197. doi: http://dx.doi.org/10.26864/PCS.v11.n1.8 